\title{
Synthesis, antitumor evaluation and molecular modeling study of novel benzimidazoles and pyrazinobenzimidazoles
}

\author{
Ahmed A. B. Mohamed ${ }^{a}$, Farid A. Badria ${ }^{\mathrm{b}}$, Azza R. Maarouf ${ }^{\mathrm{a}}$, Naglaa I. Abdel-Aziz ${ }^{\mathrm{a}^{*}}$, Fardous ElSenduny ${ }^{\mathrm{c}}$, \\ Alaa A.M. Abdel-Aziz ${ }^{\mathrm{a}, \mathrm{d}}$, Said M. Bayomi ${ }^{\mathrm{a}}$ \\ ${ }^{\mathrm{a} D}$ Department of Medicinal Chemistry, Faculty of Pharmacy, University of Mansoura, Mansoura 35516, Egypt. ${ }^{\mathrm{b}}$ Department of Pharmacognosy, Faculty of \\ Pharmacy, University of Mansoura, Mansoura 35516, Egypt. 'Department of Biochemistry, Faculty of Science, University of Mansoura, Mansoura, Egypt. \\ ${ }^{\mathrm{d}}$ Department of Pharmaceutical Chemistry, College of Pharmacy, King Saud University, Riyadh 11451, Saudi Arabia.
}

\author{
ARTICLE INFO \\ Article history: \\ Received on: 18/01/2017 \\ Accepted on: 01/03/2017 \\ Available online: 30/06/2017 \\ Key words: \\ Prop-2-en-1 - \\ one.Benzimidazoles. \\ Synthesis. Antitumor effect. \\ Molecular modelling.
}

\begin{abstract}
Some novel benzimidazole and pyrazinobenzimidazole derivatives 5-8 was designed for evaluation of their in vitro cytotoxicity studies using MTT-based assay against three cancer cell lines namely, human hepatoma cell line (HepG2), human breast cancer cell line (MCF-7) and kidney of African green monkey (Vero B). Compounds 5a and $\mathbf{5 c - e}$ exhibit the highest and broad spectrum activities against all of the three cell lines tested when compared with reference drug 5-Fluorouracil (5-FU). 1-(1H-Benzimidazol-2-yl)-3-phenylprop-2-en-1-one 5a showed superior and great potency and lethal effect against HepG2, MCF-7 and Vero B cell lines with $\mathrm{IC}_{50}$ values of $2,1.8$ and $3.5 \mu \mathrm{g} / \mathrm{ml}$, respectively, comparable to $5-\mathrm{FU}\left(\mathrm{IC}_{50}\right.$ values of 62,12 and $13 \mu \mathrm{g} / \mathrm{ml}$, respectively). Moreover, compound $\mathbf{7 b}$ showed potent activity against MCF-7 and Vero B cell lines with $\mathrm{IC}_{50}$ values of 2 and $2.5 \mu \mathrm{g} / \mathrm{ml}$, respectively. Docking study of compounds $5 \mathrm{a}$ and $7 \mathrm{~b}$ into the ATP binding site of epidermal growth factor receptor (EGFR) revealed comparable binding manner to an EGFR inhibitor, Erlotinib.
\end{abstract}

\section{INTRODUCTION}

Cancer is the result of uncoordinated and uncontrolled growth of cells. The major cause of death from cancer is metastasis (Vogelstein et al., 2013, Su et al., 2015)where cancer cells detach themselves from the parent neoplasm, invade the circulation system and spread to other body sites through several pathways (Bagi, 2002, Balmer and Valley, 2002). Cancer is continuing to be a major threat to public health and the severity of the problem, led to an impressive progress in discovering potent anticancer agents. In the scope of recognising different chemical compounds which may act as a lead for designing new antitumor agents, we are interested in this study with benzimidazole derivatives. Considerable attention has been focused on benzimidazole family as one of the bioactive heterocyclic compounds that exhibited a range of biological

* Corresponding Author

Email: naglaabdalaziz2005 @ yahoo.com and clinical applications. They are structural isosters of purine based nucleic acids and can interact with biological macromolecules such as protein, enzymes and receptors (Bansal and Silakari, 2012). Thus benzimidazole nucleus has been confirmed as an important pharmacophore in drug discovery of new antitumor agents (Hida et al., 1994, Neff et al., 2007, Styskala et al., 2008, Shaharyar et al., 2010, Hranjec et al., 2011, AbdelMohsen et al., 2010, Rahman and Siddiqui, 2010, Luo et al., 2011, Ramla et al., 2007, Ng et al., 2007, Zhong et al., 2009, Refaat, 2010, Ramla et al., 2006, Thimmegowda et al., 2008, Gowda et al., 2009). It is well known that small molecules such as pyridopyrimidines (Cockerill, et al., 2001), aminoquinazolines (ElAzab et al., 2010, Hamed et al., 2013) and benzimidazoles (Soni et al., 2012, Boschelli et al., 1999) are potent epidermal growth factor receptor-tyrosine kinase (EGFR-TK) inhibitors. Tyrosine kinase is an enzyme that can transfer a phosphate group from ATP to a protein and this is an important mechanism in regulating cell activity such as cell division. The epidermal growth factor receptors (EGFR) are over-expressed in a considerable number of human cancer cells (Cockerill and Lackey, 2002, Wakeling, 2002). 
Therefore, inhibition of EGFR-TK represented a rational approach to cancer therapy. In view of the previous findings, our research aimed at the synthesis of different substituted benzimidazolechalcones and pyrazinobenzimidazole derivatives in an attempt to reach an active antitumor agent with potentiated activity and selectivity toward cancerous cells. Computer docking methodology plays an effective role in the drug design and in the mechanistic examination by putting a molecule into the binding site of the target receptor in a noncovalent manner (Kontoyianni et al., 2004). So, the most active compounds were docked into the ATP binding site of EGFR to estimate if these compounds have comparable binding manner to an EGFR inhibitor, Erlotinib (Stamos et al., 2002).

\section{EXPERIMENTAL PROTOCOLS}

\section{Chemistry}

Melting points were recorded using Fisher-Johns apparatus and are uncorrected. IR spectra $(\mathrm{KBr})$ were determined on Mattson 5000 FT-IR spectrometer. Proton magnetic resonance $\left({ }^{1} \mathrm{HNMR}\right)$ spectra were recorded on FT-NMR spectrometer (200 $\mathrm{MHz}$ ) Gemini Varian using $\mathrm{DMSOd}_{6}$ relative to tetramethylsilane (TMS) as internal standard (chemical shifts in $\delta$ ppm). Mass spectra (MS) were measured on JEOL JMS-600H spectrometer. Elemental analysis was carried out for $\mathrm{C}, \mathrm{H}$ and $\mathrm{N}$ at the Microanalytical Centre of Cairo University. All reagents were purchased from the Aldrich Chemical Company.

The well-known compounds, 2-(1-hydroxyethyl)- $1 H$ benzimidazole (2) (Pool et al., 1937) and 2-acetyl-1Hbenzimidazole (3) (Selvakumar et al., 2012) were prepared according to the reported procedures.

General procedure for synthesis of 1-(1H-benzimidazol-2-yl)-3arylprop-2-en-1-ones (5a-f)(Scheme 1)

A mixture of 2-acetyl benzimidazole $3(1.6 \mathrm{~g}, 0.01 \mathrm{~mol})$ and the appropriate aldehyde $\mathbf{4 a - f}(0.01 \mathrm{~mol})$ in ethanol $(30 \mathrm{ml})$ and water $(30 \mathrm{ml})$ was stirred for 2 hours. Then $10 \%$ sodium hydroxide (3 $\mathrm{ml})$ was added drop-wise to the reaction mixture with continuous stirring for further 6 hours and the separated solid was filtered, dried and crystallized from aqueous ethanol to give compounds (5a-f).

\section{1-(1H-Benzimidazol-2-yl)-3-phenylprop-2-en-1-one(5a)}

yellow powder; Yield 92\%; mp 193-194 ${ }^{\circ} \mathrm{C}$; ${ }^{1} \mathrm{H}$ NMR $\left(\mathrm{DMSOd}_{6}, 200 \mathrm{MHz}\right): \delta=13.66\left(1 \mathrm{H}, \mathrm{s}, \mathrm{NH}, \mathrm{D}_{2} \mathrm{O}\right.$ exchangeable $)$, 8.53-7.39 (11H, m, Ar-H, $\mathrm{HC}=\mathrm{CH})$; Anal. Calcd for $\mathrm{C}_{16} \mathrm{H}_{12} \mathrm{~N}_{2} \mathrm{O}$ (\%): C, 77.40; H, 4.87; N, 11.28. Found: C, 77.60; H, 5.00; N, 11.58 .

\section{1-(1H-Benzimidazol-2-yl)-3-(4-methoxyphenyl)prop-2-en-1-one} (5b)

White powder; Yield 93\%; mp $171-172{ }^{\circ} \mathrm{C}$; IR $v\left(\mathrm{~cm}^{-1}\right)$; $3255(\mathrm{~N}-\mathrm{H}), 1664(\mathrm{C}=\mathrm{O}) ;{ }^{1} \mathrm{H} \mathrm{NMR}\left(\mathrm{DMSOd}_{6}, 200 \mathrm{MHz}\right): \delta=$ $13.42\left(1 \mathrm{H}, \mathrm{s}, \mathrm{NH}, \mathrm{D}_{2} \mathrm{O}\right.$ exchangeable), 8.1-7.9 (3H, m, Ar-H), 7.80
$(1 \mathrm{H}, \mathrm{d}, \mathrm{C}=\mathrm{CH}, J=15 \mathrm{~Hz}), 7.50-7.37(1 \mathrm{H}, \mathrm{m}, \mathrm{Ar}-\mathrm{H}), 7.20-7.04$ $(4 \mathrm{H}, \mathrm{m}, \mathrm{Ar}-\mathrm{H}), 6.80(1 \mathrm{H}, \mathrm{d}, \mathrm{CH}=\mathrm{CH}, \mathrm{J}=15 \mathrm{~Hz}), 3.84(3 \mathrm{H}$, $\left.\left.\mathrm{s}, \mathrm{OCH}_{3}\right) ; \mathrm{MS} \mathrm{m} / \mathrm{z}(\%) 279.10\left(\mathrm{M}^{+}+1\right), 5.15\right), 278.10\left(\mathrm{M}^{+}, 11.34\right)$, 160.90 (4.12), 136.10 (12.37), 107.05 (25.77), 104 (19.07), 67 (100); Anal. Calcd for $\mathrm{C}_{17} \mathrm{H}_{14} \mathrm{~N}_{2} \mathrm{O}_{2}(\%)$ : C, 73.37; $\mathrm{H}, 5.07 ; \mathrm{N}$, 10.07. Found: C, 73.21; H, 4.88; N, 9.81.

1-(1H-Benzimidazol-2-yl)-3-(4-(trifluoromethyl)phenyl)prop-2-en1-one $(5 c)$

White powder; Yield 87\%; mp $210-211{ }^{\circ} \mathrm{C} ;{ }^{1} \mathrm{H}$ NMR $\left(\mathrm{DMSOd}_{6}, 200 \mathrm{MHz}\right): \delta=13.57\left(1 \mathrm{H}, \mathrm{s}, \mathrm{NH}, \mathrm{D}_{2} \mathrm{O}\right.$ exchangeable), 8.34-7.25 (10H, m, Ar-H, HC=CH); MS m/z (\%) $317.95\left(\mathrm{M}^{+}+2\right.$, 1.51), $316.95\left(\mathrm{M}^{+}+1,12.28\right), 316\left(\mathrm{M}^{+}, 51.77\right), 315.05\left(\mathrm{M}^{+}-1\right.$, 5.70), 287.05 (100), 174.05 (0.10), 160 (0.09), 146 (0.64), 117.15 (7.70); Anal. Calcd for $\mathrm{C}_{17} \mathrm{H}_{11} \mathrm{~F}_{3} \mathrm{~N}_{2} \mathrm{O}(\%)$ : C, 64.56; H, 3.51; N, 8.86. Found: C, 64.20; H, 3.37; N, 8.61.

1-(1H-Benzimidazol-2-yl)-3-(4-fluorophenyl)prop-2-en-1-one (5d) White powder; Yield 91\%; mp 188-189 ${ }^{\circ} \mathrm{C}$; IR $v\left(\mathrm{~cm}^{-1}\right)$; $3267(\mathrm{~N}-\mathrm{H}), 1653(\mathrm{C}=\mathrm{O})$; ${ }^{1} \mathrm{H}$ NMR $\left(\mathrm{DMSOd}_{6}, 200 \mathrm{MHz}\right): \delta=$ $13.44\left(1 \mathrm{H}, \mathrm{s}, \mathrm{NH}, \mathrm{D}_{2} \mathrm{O}\right.$ exchangeable), 8.17-6.91 (10H, m, Ar-H, $\mathrm{HC}=\mathrm{CH}) ; \mathrm{MS} \mathrm{m} / \mathrm{z}(\%) 267.95\left(\mathrm{M}^{+}+2,1.11\right), 266.95\left(\mathrm{M}^{+}+1,7.34\right)$, $265.95\left(\mathrm{M}^{+}, 38.62\right), 265.05\left(\mathrm{M}^{+}-1,2.57\right), 246$ (100), 185.05 (1.82), 158 (1.05), 148.95 (22.53), 117 (5.70); Anal. Calcd for $\mathrm{C}_{16} \mathrm{H}_{11} \mathrm{FN}_{2} \mathrm{O}(\%)$ : C, 72.17; H , 4.16; N, 10.52. Found: C, 72.37; H , 4.33; N, 10.22.

\section{1-(1H-Benzimidazol-2-yl)-3-(pyridin-3-yl)prop-2-en-1-one (5e)}

White powder; Yield 89\%; mp $168-169{ }^{\circ} \mathrm{C}$; ${ }^{1} \mathrm{H}$ NMR $\left(\mathrm{DMSOd}_{6}, 200 \mathrm{MHz}\right): \delta=13.53\left(1 \mathrm{H}, \mathrm{s}, \mathrm{NH}, \mathrm{D}_{2} \mathrm{O}\right.$ exchangeable), $9.00(1 \mathrm{H}, \mathrm{s}, \mathrm{Ar}-\mathrm{H}), 8.70(1 \mathrm{H}, \mathrm{m}, \mathrm{Ar}-\mathrm{H}), 8.30(1 \mathrm{H}, \mathrm{d}, \mathrm{CH}=\mathrm{CH}, \mathrm{J}=$ $16 \mathrm{~Hz}), 8.20-8.00(2 \mathrm{H}, \mathrm{m}, \mathrm{Ar}-\mathrm{H}), 7.9(1 \mathrm{H}, \mathrm{d}, \mathrm{CH}=\mathrm{CH}, \mathrm{J}=16 \mathrm{~Hz})$, $7.6-6.7(4 \mathrm{H}, \mathrm{m}, \mathrm{Ar}-\mathrm{H}) ; \mathrm{MS} \mathrm{m} / \mathrm{z}(\%) 251\left(\mathrm{M}^{+}+2,2.43\right), 250$ $\left(\mathrm{M}^{+}+1,8.02\right), 249\left(\mathrm{M}^{+}, 33.31\right), 248.05\left(\mathrm{M}^{+}-1,3.18\right), 158$ (1.51), 144 (1.90), 132.05 (9.34), 117.05 (5.32), 55 (100), Anal. Calcd for $\mathrm{C}_{15} \mathrm{H}_{11} \mathrm{~N}_{3} \mathrm{O}(\%)$ : C, 72.28; H, 4.45; N, 16.86. Found: C, 71.98; H, $4.21 ; \mathrm{N}, 16.79$.

\section{1-(1H-Benzimidazol-2-yl)-3-(pyridin-4-yl)prop-2-en-1-one (5f)}

White powder; Yield 83\%; mp 203-204 ${ }^{\circ} \mathrm{C}$; IR $v\left(\mathrm{~cm}^{-1}\right)$;

$3269(\mathrm{~N}-\mathrm{H}), 1672(\mathrm{C}=\mathrm{O}) ;{ }^{1} \mathrm{H}$ NMR $\left(\mathrm{DMSOd}_{6}, 200 \mathrm{MHz}\right): \delta=$ 13.27 (1H, s, NH, D $\mathrm{D}_{2}$ exchangeable), 8.80-6.95 (10H, m, Ar-H, $\mathrm{HC}=\mathrm{CH}) ; \mathrm{MS} \mathrm{m} / \mathrm{z}(\%) 250.95\left(\mathrm{M}^{+}+2,1.31\right), 249.90\left(\mathrm{M}^{+}+1,3.37\right)$, $248.95\left(\mathrm{M}^{+}, 12.86\right), 247.95\left(\mathrm{M}^{+}-1,1.20\right), 157.95(0.21), 132$ (8.70), 118 (100), 117.05 (6.10), 91 (30.49); Anal. Calcd for $\mathrm{C}_{15} \mathrm{H}_{11} \mathrm{~N}_{3} \mathrm{O}(\%)$ : C, 72.28; H, 4.45; N, 16.86. Found: C, 72.48; H, $4.25 ; \mathrm{N}, 16.99$.

General procedure for synthesis of 1-\{1-[2-(4-substitutedphenyl)2-oxoethyl]-1H-benzimidazol-2-yl\}-3-arylprop-2-en-1-ones (7a-j) (Scheme 2)

A mixture of chalcone derivatives 5a-f $(0.006 \mathrm{~mol})$, the appropriate $\alpha$-bromophenacyl derivative 6a,b $(0.006 \mathrm{~mol})$ and anhydrous potassium carbonate $(0.007 \mathrm{~mol})$ in acetone $(100 \mathrm{ml})$ 
was stirred at room temperature for 6 hours. The solvent was evaporated under reduced pressure and the residue was triturated with water and filtered. The raw product was crystallized from aqueous ethanol to give compounds $7 \mathbf{a}-\mathbf{j}$.

1-\{1-[2-(4-Chlorophenyl)-2-oxoethyl]-1H-benzimidazol-2-yl\}-3(4-methoxyphenyl)prop-2-en-1-one (7a).

White powder; Yield $84 \%$; mp $188-189{ }^{\circ} \mathrm{C}$; IR $v\left(\mathrm{~cm}^{-1}\right)$; 1692, $1660(\mathrm{C}=\mathrm{O}), 1599,1450(\mathrm{C}=\mathrm{N}, \mathrm{C}=\mathrm{C}) ;{ }^{1} \mathrm{H}$ NMR $\left(\mathrm{DMSOd}_{6}\right.$, $200 \mathrm{MHz}): \delta=8.36-6.72(14 \mathrm{H}, \mathrm{m}, \mathrm{Ar}-\mathrm{H}, \mathrm{CH}=\mathrm{CH}), 6.07(2 \mathrm{H}, \mathrm{s}$, $\left.\mathrm{CH}_{2} \mathrm{CO}\right), 3.66\left(3 \mathrm{H}, \mathrm{s}, \mathrm{OCH}_{3}\right) ; \mathrm{MS} \mathrm{m} / \mathrm{z}(\%) 432.80\left(\mathrm{M}^{+}+2,0.37\right)$, $431.80\left(\mathrm{M}^{+}+1,0.28\right), 430.24\left(\mathrm{M}^{+}, 0.24\right), 400.80(0.24), 325.10$ (1.68), 320.10 (0.36), 57.05 (100); Anal. Calcd for $\mathrm{C}_{25} \mathrm{H}_{19} \mathrm{ClN}_{2} \mathrm{O}_{3}$ (\%): C, 69.69; H, 4.44; N, 6.50. Found: C, 69.51; H,4.27; N, 6.33.

1-\{1-[2-(4-Bromophenyl)-2-oxoethyl]-1H-benzimidazol-2-yl\}-3-(4methoxyphenyl)prop-2-en-1-one (7b)

White powder; Yield 89\%; mp 201-202 ${ }^{\circ} \mathrm{C}$; ${ }^{1} \mathrm{H}$ NMR $\left(\mathrm{DMSOd}_{6}, 200 \mathrm{MHz}\right): \delta=8.33-6.58(14 \mathrm{H}, \mathrm{m}, \mathrm{Ar}-\mathrm{H}, \mathrm{CH}=\mathrm{CH})$, $5.73\left(2 \mathrm{H}, \mathrm{s}, \mathrm{CH}_{2} \mathrm{CO}\right), 3.76\left(3 \mathrm{H}, \mathrm{s}, \mathrm{OCH}_{3}\right) ; \mathrm{MS} \mathrm{m} / \mathrm{z}(\%) 475.25$ $\left(\mathrm{M}^{+}, 0.05\right), 474.10\left(\mathrm{M}^{+}-1,0.04\right), 369.15$ (1.86), 278 (3.47), 248.05 (3.71), 199 (0.92), 138.95 (100); Anal. Calcd for $\mathrm{C}_{25} \mathrm{H}_{19} \mathrm{BrN}_{2} \mathrm{O}_{3}$ (\%): C, 63.17; H, 4.03; N, 5.89. Found: C, 62.91; H, 4.21; N, 5.68 .

1-\{1-[2-(4-Chlorophenyl)-2-oxoethyl]-1H-benzimidazol-2-yl\}-3(4-(trifluoromethyl)phenyl)prop-2-en-1-one (7c)

White powder; Yield 78\%; mp 196-197 ${ }^{\circ} \mathrm{C}$; IR $v\left(\mathrm{~cm}^{-1}\right)$; 1694, $1657(\mathrm{C}=\mathrm{O}), 1595,1448(\mathrm{C}=\mathrm{N}, \mathrm{C}=\mathrm{C})$; ${ }^{1} \mathrm{H}$ NMR $\left(\mathrm{DMSOd}_{6}\right.$, $200 \mathrm{MHz}): \delta=8.32-7.22(14 \mathrm{H}, \mathrm{m}, \mathrm{Ar}-\mathrm{H}, \mathrm{CH}=\mathrm{CH}), 6.06(2 \mathrm{H}, \mathrm{s}$, $\left.\mathrm{CH}_{2} \mathrm{CO}\right) ; \mathrm{MS} \mathrm{m} / \mathrm{z}(\%) 468.30\left(\mathrm{M}^{+}, 1.14\right), 467.30\left(\mathrm{M}^{+}-1,3.42\right)$, 366.30 (1.05), 316.30 (0.55), 154.05 (1.69), 57.05 (100); Anal. Calcd for $\mathrm{C}_{25} \mathrm{H}_{16} \mathrm{ClF}_{3} \mathrm{~N}_{2} \mathrm{O}_{2}$ (\%): C, 64.04; H, 3.44; N, 5.97. Found: C, 63.91; H, 3.40; N, 5.78.

1-\{1-[2-(4-Bromophenyl)-2-oxoethyl]-1H-benzimidazol-2-yl\}-3-(4(trifluoromethyl)phenyl)prop-2-en-1-one (7d)

White powder; Yield 80\%; mp 246-247 ${ }^{\circ} \mathrm{C}$; ${ }^{1} \mathrm{H}$ NMR $\left(\mathrm{DMSOd}_{6}, 200 \mathrm{MHz}\right): \delta=7.93-7.06(12 \mathrm{H}, \mathrm{m}, \mathrm{Ar}-\mathrm{H}), 7.84(1 \mathrm{H}, \mathrm{d}$, $\mathrm{J}=16 \mathrm{~Hz}), 7.08(1 \mathrm{H}, \mathrm{d}, \mathrm{J}=16 \mathrm{~Hz}), 6.12\left(2 \mathrm{H}, \mathrm{s}, \mathrm{CH}_{2} \mathrm{CO}\right) ; \mathrm{MS} \mathrm{m} / \mathrm{z}$ (\%) $514.95\left(\mathrm{M}^{+}+2,2.74\right), 513.95\left(\mathrm{M}^{+}+1,9.20\right), 512.95\left(\mathrm{M}^{+}, 8.77\right)$, $511.90\left(\mathrm{M}^{+}-1,26.90\right), 395$ (3.84), 369 (1.87), 197.95 (2.69), 182.90 (100), 116.05 (5.75); Anal. Calcd for $\mathrm{C}_{25} \mathrm{H}_{16} \mathrm{BrF}_{3} \mathrm{~N}_{2} \mathrm{O}_{2}(\%)$ : C, 58.50; H, 3.14; N, 5.46. Found: C, 58.21; H, 3.07; N, 5.66.

\section{1-\{1-[2-(4-Chlorophenyl)-2-oxoethyl]-1H-benzimidazol-2-yl\}-3-}

(4-fluorophenyl)prop-2-en-1-one (7e)

White powder; Yield 86\%; mp 231-232 ${ }^{\circ} \mathrm{C} ;{ }^{1} \mathrm{H}$ NMR $\left(\mathrm{DMSOd}_{6}, 200 \mathrm{MHz}\right): \delta=7.98-6.88(14 \mathrm{H}, \mathrm{m}, \mathrm{Ar}-\mathrm{H}$, $\mathrm{CH}=\mathrm{CH}), \quad 6.22\left(\mathrm{~s}, \quad 2 \mathrm{H}, \quad \mathrm{s}, \quad \mathrm{CH}_{2} \mathrm{CO}\right) ; \mathrm{MS} \mathrm{m} / \mathrm{z} \quad(\%) 419.10$ $\left(\mathrm{M}^{+}+1,4.07\right), 418.20\left(\mathrm{M}^{+}, 4.07\right), 400.20$ (0.38), 290 (0.62), 267.20 (0.62), 69.05 (100); Anal. Calcd for $\mathrm{C}_{24} \mathrm{H}_{16} \mathrm{ClFN}_{2} \mathrm{O}_{2}(\%)$ : C, 68.82; H, 3.85; N, 6.69. Found: C, 69.02; H, 3.45; N, 6.31.
1-\{1-[2-(4-Bromophenyl)-2-oxoethyl]-1H-benzimidazol-2-yl\}-3-(4fluorophenyl)prop-2-en-1-one (7f)

White powder; Yield 81\%; $\mathrm{mp} \quad 256-257 \quad{ }^{\circ} \mathrm{C}$; IR $v\left(\mathrm{~cm}^{-1}\right) ; 1693,1655 \quad(\mathrm{C}=\mathrm{O}), \quad 1599,1450 \quad(\mathrm{C}=\mathrm{N}, \quad \mathrm{C}=\mathrm{C})$; ${ }^{1} \mathrm{H}$ NMR $\left(\mathrm{DMSOd}_{6}, 200 \mathrm{MHz}\right): \delta=8.18-6.93(12 \mathrm{H}, \mathrm{m}, \mathrm{Ar}-\mathrm{H})$, $7.22(1 \mathrm{H}, \mathrm{d}, \mathrm{CH}=\mathrm{CH}, \mathrm{J}=15 \mathrm{~Hz}), 7.05(1 \mathrm{H}, \mathrm{d}, \mathrm{CH}=\mathrm{CH}, \mathrm{J}=15 \mathrm{~Hz})$, $5.66\left(2 \mathrm{H}, \mathrm{s}, \mathrm{CH}_{2} \mathrm{CO}\right) ; \mathrm{MS} \mathrm{m} / \mathrm{z}(\%) 463.80\left(\mathrm{M}^{+}+1,1.51\right)$, $462.80\left(\mathrm{M}^{+}, 0.82\right), 461.90\left(\mathrm{M}^{+}-1,1.69\right), 307.90$ (1.89), 265.05 (8.12), 149.05 (15.79), 118.05 (100); Anal. Calcd for $\mathrm{C}_{24} \mathrm{H}_{16} \mathrm{BrFN}_{2} \mathrm{O}_{2}(\%)$ : C, 62.22; H, 3.48; N, 6.05. Found: C, 62.40; $\mathrm{H}, 3.21$; N, 6.39 .

1-\{1-[2-(4-Chlorophenyl)-2-oxoethyl]-1H-benzimidazol-2-yl\}-3(pyridin-3-yl)prop-2-en-1-one (7g)

White powder; Yield 81\%; mp $175-176{ }^{\circ} \mathrm{C}$; ${ }^{1} \mathrm{H}$ NMR $\left(\mathrm{DMSOd}_{6}, 200 \mathrm{MHz}\right): \delta=8.62-6.91(14 \mathrm{H}, \mathrm{m}, \mathrm{Ar}-\mathrm{H}, \mathrm{CH}=\mathrm{CH})$, $6.28\left(2 \mathrm{H}, \mathrm{s}, \mathrm{CH}_{2} \mathrm{CO}\right) ; \mathrm{MS} \mathrm{m} / \mathrm{z}(\%) 404.20\left(\mathrm{M}^{+}+2,0.08\right), 403.20$ $\left(\mathrm{M}^{+}+1,0.14\right), 402.20\left(\mathrm{M}^{+}, 0.17\right), 401.20\left(\mathrm{M}^{+}-1,0.17\right), 366.85$ (0.63), 324.90 (0.09), 290.90 (0.20), 105 (100); Anal. Calcd for $\mathrm{C}_{23} \mathrm{H}_{16} \mathrm{ClN}_{3} \mathrm{O}_{2}(\%)$ : C, 68.74; H, 4.01; N, 10.46. Found: C, 68.53; H, 4.39; N, 10.18.

1-\{1-[2-(4-Bromophenyl)-2-oxoethyl]-1H-benzimidazol-2-yl\}-3(pyridin-3-yl)prop-2-en-1-one (7h)

White powder; Yield 86\%; mp 267-268 ${ }^{\circ} \mathrm{C}$; ${ }^{1} \mathrm{H}$ NMR $\left(\mathrm{DMSOd}_{6}, 200 \mathrm{MHz}\right): \delta=7.91-6.92(\mathrm{~m}, 12 \mathrm{H}, \mathrm{m}, \mathrm{Ar}-\mathrm{H}), 7.78(1 \mathrm{H}$, $\mathrm{d}, \mathrm{CH}=\mathrm{CH}, \mathrm{J}=16 \mathrm{~Hz}), 7.08(1 \mathrm{H}, \mathrm{CH}=\mathrm{CH}, \mathrm{d}, \mathrm{J}=16 \mathrm{~Hz}), 6.27(2 \mathrm{H}$, $\left.\mathrm{s}, \mathrm{CH}_{2} \mathrm{CO}\right) ; \mathrm{MS} \mathrm{m} / \mathrm{z}(\%) 447.80\left(\mathrm{M}^{+}+2,3.04\right), 446.80\left(\mathrm{M}^{+}+1\right.$, 3.61), $445.80 \quad\left(\mathrm{M}^{+}, \quad 2.92\right), \quad 369.10 \quad(0.16), \quad 291.10 \quad(0.40)$, 249.10 (0.12), 1.85 (3.81), 105 (100); Anal. Calcd for $\mathrm{C}_{23} \mathrm{H}_{16} \mathrm{BrN}_{3} \mathrm{O}_{2}(\%)$ : C, 61.90; H, 3.61; N, 9.42. Found: C, 62.03; H, 3.30; N, 9.78 .

1-\{1-[2-(4-Chlorophenyl)-2-oxoethyl]-1H-benzimidazol-2-yl\}-3(pyridin-4-yl)prop-2-en-1-one (7i)

White powder; Yield 79\%; mp 228-229 ${ }^{\circ} \mathrm{C} ; \mathrm{v}\left(\mathrm{cm}^{-1}\right)$; 1691, $1657(\mathrm{C}=\mathrm{O}), 1563,1453(\mathrm{C}=\mathrm{N}, \mathrm{C}=\mathrm{C})$; ${ }^{1} \mathrm{H}$ NMR $\left(\mathrm{DMSOd}_{6}\right.$, $200 \mathrm{MHz}): \delta=8.49-6.82(14 \mathrm{H}, \mathrm{m}, \mathrm{Ar}-\mathrm{H}, \mathrm{CH}=\mathrm{CH}), 6.29(2 \mathrm{H}, \mathrm{s}$, $\left.\mathrm{CH}_{2} \mathrm{CO}\right) ; \mathrm{MS} \mathrm{m} / \mathrm{z}(\%) 404.10\left(\mathrm{M}^{+}+2,0.51\right), 403.10\left(\mathrm{M}^{+}+1,0.43\right)$, $402.10\left(\mathrm{M}^{+}, 0.74\right), 401.10\left(\mathrm{M}^{+}-1,0.36\right), 367.10$ (0.58), 325.10 (0.61), 291.10 (0.84), 55 (100); Anal. Calcd for $\mathrm{C}_{23} \mathrm{H}_{16} \mathrm{ClN}_{3} \mathrm{O}_{2}$ (\%): C, 68.74; H, 4.01; N, 10.46. Found: C, 68.66; H, 3.77; N, 10.23 .

1-\{1-[2-(4-Bromophenyl)-2-oxoethyl]-1H-benzimidazol-2-yl\}-3(pyridin-4-yl)prop-2-en-1-one (7j)

White powder; Yield 84\%; mp 278-279 ${ }^{\circ} \mathrm{C}$; ${ }^{1} \mathrm{H}$ NMR $\left(\mathrm{DMSOd}_{6}, 200 \mathrm{MHz}\right): \delta=8.46-6.96(12 \mathrm{H}, \mathrm{m}, \mathrm{Ar}-\mathrm{H}), 7.94(1 \mathrm{H}, \mathrm{d}$, $\mathrm{CH}=\mathrm{CH}, \mathrm{J}=17 \mathrm{~Hz}), 7.38(1 \mathrm{H}, \mathrm{d}, \mathrm{CH}=\mathrm{CH}, \mathrm{J}=17 \mathrm{~Hz}), 6.30(2 \mathrm{H}, \mathrm{s}$, $\left.\mathrm{CH}_{2} \mathrm{CO}\right) ; \mathrm{MS} \mathrm{m} / \mathrm{z}(\%) 446.90\left(\mathrm{M}^{+}+1,0.94\right), 445.90\left(\mathrm{M}^{+}, 0.72\right)$, $444.90\left(\mathrm{M}^{+}-1,0.86\right), 316.10$ (0.89), 248.10 (0.83), 133.10 (3.55), 55 (100); Anal. Calcd for $\mathrm{C}_{23} \mathrm{H}_{16} \mathrm{BrN}_{3} \mathrm{O}_{2}(\%)$ : C, 61.90; H, 3.61; N, 9.42. Found: C, 62.16; H, 3.97; N, 9.23. 
General procedure for synthesis of 1-(2-arylvinyl)-3-(4substitutedphenyl)pyrazino [1,2-a]benzimidazole derivatives $(8 a-j)$ (Scheme 2)

A mixture of compound $\mathbf{7 a - j}(0.003 \mathrm{~mol})$ and ammonium acetate $(0.03 \mathrm{~mol})$ in $50 \mathrm{ml}$ of acetic acid was refluxed for 1 hour. The reaction mixture was cooled, poured into ice water and neutralized with sodium carbonate solution. The formed precipitate was filtered, dried and crystallized from aqueous ethanol to give compounds $\mathbf{8 a - j}$.

1-(2-(4-Methoxyphenyl)vinyl)-3-(4-chlorophenyl)pyrazino[1,2a]benzimidazole $(8 a)$

White powder; Yield 74\%; mp 198-199 ${ }^{\circ} \mathrm{C}$; IR $v\left(\mathrm{~cm}^{-1}\right)$; 1630, $1453(\mathrm{C}=\mathrm{N}, \mathrm{C}=\mathrm{C}) ;{ }^{1} \mathrm{H}$ NMR $\left(\mathrm{DMSOd}_{6}, 200 \mathrm{MHz}\right): \delta=8.82$ $(1 \mathrm{H}, \mathrm{s}, \mathrm{Ar}-\mathrm{H}), 8.18-6.68(14 \mathrm{H}, \mathrm{m}, \mathrm{Ar}-\mathrm{H}, \mathrm{CH}=\mathrm{CH}), 3.46(3 \mathrm{H}, \mathrm{s}$, $\left.\mathrm{OCH}_{3}\right) ; \mathrm{MS} \mathrm{m} / \mathrm{z}(\%) 413.20\left(\mathrm{M}^{+}+2,0.32\right), 412.20\left(\mathrm{M}^{+}+1,0.41\right)$, $411.20\left(\mathrm{M}^{+}, 0.86\right), 410.20\left(\mathrm{M}^{+}-1,0.32\right), 381.20(0.74), 306.10$ (0.67), 301.10 (0.57), 57.05 (100); Anal. Calcd for $\mathrm{C}_{25} \mathrm{H}_{18} \mathrm{ClN}_{3} \mathrm{O}$ (\%): C, 72.90; H, 4.40; N, 10.20. Found: C, 73.16; H, 4.07; N, 9.98 .

1-[2-(4-Methoxyphenyl)vinyl]-3-(4-bromophenyl)pyrazino[1,2a]benzimidazole $(\mathbf{8 b})$

White powder; Yield 79\%; mp $186-187{ }^{\circ} \mathrm{C}$; ${ }^{1} \mathrm{H}$ NMR $\left(\mathrm{DMSOd}_{6}, 200 \mathrm{MHz}\right): \delta=8.72(1 \mathrm{H}, \mathrm{s}, \mathrm{Ar}-\mathrm{H}), 8.36-6.72(14 \mathrm{H}, \mathrm{m}$, $\mathrm{Ar}-\mathrm{H}, \mathrm{CH}=\mathrm{CH}), 3.64\left(3 \mathrm{H}, \mathrm{s}, \mathrm{OCH}_{3}\right) ; \mathrm{MS} \mathrm{m} / \mathrm{z}(\%) 458\left(\mathrm{M}^{+}+2\right.$, 6.62), $457\left(\mathrm{M}^{+}+1,17.25\right), 455.95\left(\mathrm{M}^{+}, 42.16\right), 454.95\left(\mathrm{M}^{+}-1\right.$, 39.72), 377 (1.39), 352.05 (1.57), 301 (30.14), 63 (100); Anal. Calcd for $\mathrm{C}_{25} \mathrm{H}_{18} \mathrm{BrN}_{3} \mathrm{O}(\%)$ : C, 65.80; H, 3.98; N, 9.21. Found: $\mathrm{C}$, 65.69; H, 3.81; N, 9.07.

1-[2-(4-Trifluoromethylphenyl)vinyl]-3-(4-chlorophenyl)pyrazino [1,2-a]benzimidazole $(8 c)$

White powder; Yield 81\%; mp 213-214 ${ }^{\circ} \mathrm{C} ; v\left(\mathrm{~cm}^{-1}\right)$; $1631,1446(\mathrm{C}=\mathrm{N}, \mathrm{C}=\mathrm{C}) ;{ }^{1} \mathrm{H}$ NMR $\left(\mathrm{DMSOd}_{6}, 200 \mathrm{MHz}\right): \delta=8.68$ $(1 \mathrm{H}, \mathrm{s}, \mathrm{Ar}-\mathrm{H}), 8.16-7.18(14 \mathrm{H}, \mathrm{m}, \mathrm{Ar}-\mathrm{H}, \mathrm{CH}=\mathrm{CH}) ; \mathrm{MS} \mathrm{m} / \mathrm{z}(\%)$ $451.90\left(\mathrm{M}^{+}+2,7.74\right), 450.59\left(\mathrm{M}^{+}+1,6.75\right), 449.90\left(\mathrm{M}^{+}, 19.38\right)$, $448.95\left(\mathrm{M}^{+}-1,2.40\right), 415.20$ (0.51), 339.10 (1.60), $305.10(0.41)$, 57 (100);Anal. Calcd for $\mathrm{C}_{25} \mathrm{H}_{15} \mathrm{ClF}_{3} \mathrm{~N}_{3}(\%)$ : C, 66.75; H, 3.36; N, 9.34. Found: C, 66.37; H, 3.01; N, 9.12.

1-[2-(4-Trifluoromethylphenyl)vinyl]-3-(4-bromophenyl)pyrazino [1,2-a]benzimidazole $(\mathbf{8 d})$

White powder; Yield 84\%; mp 233-234 ${ }^{\circ} \mathrm{C}$; ${ }^{1} \mathrm{H}$ NMR $\left(\mathrm{DMSOd}_{6}, 200 \mathrm{MHz}\right): \delta=8.81(1 \mathrm{H}, \mathrm{s}, \mathrm{Ar}-\mathrm{H}), 7.85-7.12(14 \mathrm{H}, \mathrm{m}$, $\mathrm{Ar}-\mathrm{H}, \mathrm{CH}=\mathrm{CH}) ; \mathrm{MS} \mathrm{m} / \mathrm{z}(\%) 494.10\left(\mathrm{M}^{+}, 22.55\right), 493.10\left(\mathrm{M}^{+}-1\right.$, 67.65), 464.10 (72.55), 324.10 (76.47), 291.10 (64.71), 167.10 (100); Anal. Calcd for $\mathrm{C}_{25} \mathrm{H}_{15} \mathrm{BrF}_{3} \mathrm{~N}_{3}(\%)$ : C, 60.75; H, 3.06; N, 8.50. Found: C, 61.02; H, 3.02; N, 8.02.

1-[2-(4-Fluorophenyl)vinyl]-3- (4-chlorophenyl) pyrazino[1,2a]benzimidazole $(\mathbf{8 e})$

White powder; Yield 80\%; mp 207-208 ${ }^{\circ} \mathrm{C}$; ${ }^{1} \mathrm{H}$ NMR $\left(\mathrm{DMSOd}_{6}, 200 \mathrm{MHz}\right): \delta=8.76(1 \mathrm{H}, \mathrm{s}, \mathrm{Ar}-\mathrm{H}), 8.24-6.93(14 \mathrm{H}, \mathrm{m}$,
Ar-H, $\quad \mathrm{CH}=\mathrm{CH}) ; \quad \mathrm{MS} \mathrm{m} / \mathrm{z}(\%) 401.20\left(\mathrm{M}^{+}+2, \quad 0.25\right), \quad 400.20$

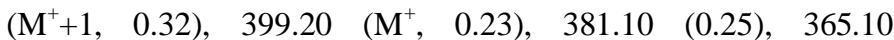
(0.26), 289.10 (2.13), 55 (100); Anal. Calcd for $\mathrm{C}_{24} \mathrm{H}_{15} \mathrm{ClFN}_{3}$ (\%): C, 72.09; H, 3.78; N, 10.51. Found: C, 71.97; H, 3.67; N, 10.38 .

1-[2-(4-Fluorophenyl)vinyl]-3-(4-bromophenyl)pyrazino[1,2a]benzimidazole $(8 f)$

White powder; Yield $76 \%$; mp $168-169{ }^{\circ} \mathrm{C} ; v\left(\mathrm{~cm}^{-1}\right)$; $1630,1454(\mathrm{C}=\mathrm{N}, \mathrm{C}=\mathrm{C}) ;{ }^{1} \mathrm{H} \quad \mathrm{NMR} \quad\left(\mathrm{DMSOd}_{6}, 200 \mathrm{MHz}\right)$ : $\delta=8.92(1 \mathrm{H}, \mathrm{s}, \mathrm{Ar}-\mathrm{H}), 8.18-6.83(14 \mathrm{H}, \mathrm{m}, \mathrm{Ar}-\mathrm{H}, \mathrm{CH}=\mathrm{CH}) ; \mathrm{MS}$ $\mathrm{m} / \mathrm{z} \quad(\%) 445.90 \quad\left(\mathrm{M}^{+}+2,0.42\right), 444.90 \quad\left(\mathrm{M}^{+}+1,0.51\right), 443.90$

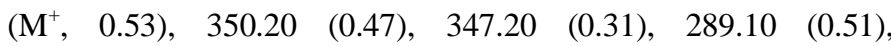
169.10 (1.78), 122.10 (4.06), 57 (100); Anal. Calcd for $\mathrm{C}_{24} \mathrm{H}_{15} \mathrm{BrFN}_{3}(\%)$ : C, 64.88; H, 3.40; N, 9.46. Found: C, 64.66; H, $3.67 ; \mathrm{N}, 9.18$.

1-[2-(3-Pyridyl)vinyl]-3-(4-chlorophenyl)pyrazino[1,2-

a]benzimidazole $\mathbf{( 8 g})$

White powder; Yield 69\%; mp 236-237 ${ }^{\circ} \mathrm{C}$; ${ }^{1} \mathrm{H}$ NMR $\left(\mathrm{DMSOd}_{6}, 200 \mathrm{MHz}\right): \delta=8.62(1 \mathrm{H}, \mathrm{s}, \mathrm{Ar}-\mathrm{H}), 8.32-6.94(14 \mathrm{H}, \mathrm{m}$, Ar-H, CH=CH); MS m/z (\%) $384.30\left(\mathrm{M}^{+}+2,0.58\right), 383.20\left(\mathrm{M}^{+}+1\right.$, 1.55), $382.30\left(\mathrm{M}^{+}, 2.03\right), 381.15\left(\mathrm{M}^{+}-1,2.39\right), 350.20(0.42)$, 293.20 (0.71), 195 (1.57), 57 (100)' Anal. Calcd for $\mathrm{C}_{23} \mathrm{H}_{15} \mathrm{ClN}_{4}$ (\%): C, 72.16; H, 3.95; N, 14.63. Found: C, 72.46; H, 3.67; N, 15.00 .

1-[2-(3-Pyridyl)vinyl]-3-(4-bromophenyl)pyrazino[1,2-

a]benzimidazole $(\mathbf{8 h})$

White powder; Yield 73\%; mp 223-224 ${ }^{\circ} \mathrm{C}$; ${ }^{1} \mathrm{H}$ NMR $\left(\mathrm{DMSOd}_{6}, 200 \mathrm{MHz}\right): \delta=8.64(1 \mathrm{H}, \mathrm{s}, \mathrm{Ar}-\mathrm{H}), 6.94-8.12(14 \mathrm{H}, \mathrm{m}$, $\mathrm{Ar}-\mathrm{H}, \mathrm{CH}=\mathrm{CH}) ; \mathrm{MS} \mathrm{m} / \mathrm{z}(\%) 428.20\left(\mathrm{M}^{+}+1,0.10\right), 427.20\left(\mathrm{M}^{+}\right.$, 0.08), $426.20\left(\mathrm{M}^{+}-1,0.09\right), 351.20$ (0.09), 272.10 (0.16), 195.10 (0.43), 69.05 (100)' Anal. Calcd for $\mathrm{C}_{23} \mathrm{H}_{15} \mathrm{BrN}_{4}(\%)$ : C, 64.65; H, 3.54; N, 13.11. Found: C, 64.38; H, 3.37; N, 13.01.

\section{1-[2-(4-Pyridyl)vinyl]-3-(4-chlorophenyl)pyrazino[1,2- a]benzimidazole (8i)}

White powder; Yield 80\%; mp 218-219 ${ }^{\circ} \mathrm{C}$; IRv $\left(\mathrm{cm}^{-1}\right)$; $1630,1450(\mathrm{C}=\mathrm{N}, \mathrm{C}=\mathrm{C}) ;{ }^{1} \mathrm{H}$ NMR $\left(\mathrm{DMSOd}_{6}, 200 \mathrm{MHz}\right): \delta=8.67$ (1H, s, Ar-H), 8.18-7.11 (14H, m, Ar-H, CH=CH); MS m/z (\%) $383.10\left(\mathrm{M}^{+}+1,62.25\right), 382.10\left(\mathrm{M}^{+}, 58.16\right), 349.10$ (2.13), 271.10 (18.44), 244.10 (7.80), 60 (100); Anal. Calcd for $\mathrm{C}_{23} \mathrm{H}_{15} \mathrm{ClN}_{4}(\%)$ : C, 72.16; H, 3.95; N, 14.63. Found: C, 72.38; H, $3.57 ; \mathrm{N}, 14.22$.

\section{1-[2-(4-Pyridyl)vinyl]-3-(4-bromophenyl)pyrazino[1,2-}

a]benzimidazole $(8 \mathrm{j})$

White powder; Yield 72\%; mp 243-244 ${ }^{\circ} \mathrm{C}$; ${ }^{1} \mathrm{H}$ NMR $\left(\mathrm{DMSOd}_{6}, 200 \mathrm{MHz}\right): \delta=8.68(1 \mathrm{H}, \mathrm{s}, \mathrm{Ar}-\mathrm{H}), 8.50-6.80(14 \mathrm{H}, \mathrm{m}$, $\mathrm{Ar}-\mathrm{H}, \mathrm{CH}=\mathrm{CH}) ; \mathrm{MS} \mathrm{m} / \mathrm{z}(\%) ; 428.90\left(\mathrm{M}^{+}+1,39.51\right), 427.80\left(\mathrm{M}^{+}\right.$, 44.70), $426.90\left(\mathrm{M}^{+}-1,47.80\right), 370.20$ (45.85), 254.10 (85.37), 192.10 (42.39), 127.10 (100); Anal. Calcd for $\mathrm{C}_{23} \mathrm{H}_{15} \mathrm{BrN}_{4}(\%): \mathrm{C}$, 64.65; H, 3.54; N, 13.11. Found: C, 64.35; H, 3.42; N, 12.88. 


\section{BIOLOGICAL EVALUATION}

\section{Cytotoxicity screening}

The cytotoxicity screening was done by employing tetrazolium salt MTT assay (Mosmann et al., 1983, Denizot and Lang, 1986).Serial dilutions $(60 \mu \mathrm{L})$ of the test compounds and 5florouracil dissolved in $0.05 \%$ DMSO were given to $120 \mu \mathrm{L}$ of the suspended cells $(50,000$ cells $/ \mathrm{mL})$ in wells of 96 -well plates. The viability of the cells was measured by the colorimetric MTT assay after 5 days of incubation according to the reported procedures. The absorbance of the purple formazan solution was measured at $\lambda_{540} \mathrm{~nm}$ by microplate reader (ELx800 Absorbance Microplate Reader, BioTek) against DMSO as a negative control. The cytotoxicity of the tested compounds was expressed as the concentration that resulted in a $50 \%$ inhibition of growth $\left(\mathrm{IC}_{50}\right)$ compared to the untreated cells (DMSO without the tested compounds) and 5- FU. Qualitative morphological study by MTT assay was done for compounds $\mathbf{5 c}$ and $\mathbf{7 i}$ against MCF-7 and HepG2, respectively using high dose of $37 \mu \mathrm{g} / \mathrm{ml}$ and low dose of $4 \mu \mathrm{g} / \mathrm{ml}$ for compound $\mathbf{5 c}$ and doses of 37, 12 and $4 \mu \mathrm{g} / \mathrm{ml}$ for compound 7i. Images were taken using $\mathrm{Gx}$ microscope (GXMGXD202 Inverted Microscope) (10x Eyepiece) and DMSO was used as a negative control.

\section{MOLECULAR DOCKING METHODOLOGY}

Docking studies have been carried out using MOE 2008.10 (MOE 2008.10 of Chemical Computing Group. Inc.).The crystal structure of EGFR with Erlotinib (Tarceva ${ }^{\mathrm{TM}}$ ) (PDB code: 1M17) were obtained from protein data bank (PDB). Docking study was proceeded according to the standard official procedure of MOE 2008.10 (MOE 2008.10 of Chemical Computing Group. Inc, Halgren, 1996)and the MOE's Pose Viewer utility (El-Azab et al., 2010).

\section{RESULTS and DISCUSSION}

\section{Chemistry}

The reaction sequence used to synthesize the desired compounds is depicted in Schemes 1 and 2.

\section{Synthesis of compounds 2-5 (Scheme 1)}

The reported 2-(1-hydroxyethyl)- $1 H$ - benzimidazole 2 was obtained in a high yield via cyclization of $o$-phenylenediamine with lactic acid in $4 \mathrm{~N}-\mathrm{HCl}$. Oxidation of 2-(1-hydroxyethyl)- $1 \mathrm{H}$ benzimidazole $\mathbf{2}$ with potassium dichromate in concentrated sulphuric acid gave 2-acetyl- $1 H$-benzimidazole 3 . Claisen-Schmidt condensation is the most convenient method for preparation of $\alpha, \beta$-unsaturated carbonyl compounds in which an equimolar quantity of aromatic aldehyde and aliphatic aldehyde or ketone was condensed in the presence of $10-60 \%$ aqueous alcoholic alkali. Hence, condensation of 2-acetyl- $1 H$ - benzimidazole 3 and a variety of aromatic aldehydes $\mathbf{4 a - f}$ in aqueous ethanolic solution of sodium hydroxide $10 \%$ gave 1-(1H-benzimidazol-2-yl)-3arylprop-2-en-1-ones 5a-f. The structures of the prepared compounds were substantiated by elemental and spectral analyses. For example, in ${ }^{1} \mathrm{H}$ NMR spectrum of compounds $\mathbf{5 b}$ and $\mathbf{5 e}$, the vinylic protons resonated as two doublets within the aromatic region with $J$ value $15 \mathrm{~Hz}$ and $16 \mathrm{~Hz}$, respectively and this observation indicated the trans configuration (Silverstein et al.,1991).

\section{Synthesis of compounds 7and 8 (Scheme 2)}

$\mathrm{N}$-Alkylation of benzimidazoles was successfully achieved via substitution reaction with alkyl halides. Elaboration to compounds $\mathbf{7 a - j}$ was performed by the reaction of compounds 5a-f with the appropriate $\alpha$-bromophenacyl derivatives $\mathbf{6 a}, \mathbf{b}$ in dry acetone in the presence of anhydrous $\mathrm{K}_{2} \mathrm{CO}_{3}$. The structures of compounds $\mathbf{7} \mathbf{a}-\mathbf{j}$ were confirmed by IR that revealed the presence of two different carbonyl bands due to propenone and oxoethyl residues at about 1660-1655 $\mathrm{cm}^{-1}$ and 1694-1691 $\mathrm{cm}^{-1}$, respectively. Also, ${ }^{1} \mathrm{HNMR}$ spectra were characterized by the presence of methylene protons which resonate in aliphatic area in the range of 6.30-5.66 ppm. Moreover, the vinylic protons appeared as two doublets within the aromatic region in the ${ }^{1} \mathrm{H}$ NMR spectra of compounds $\mathbf{7 d}, \mathbf{7 f}, \mathbf{7 h}$ and $\mathbf{7 j}$ with high values of $J$ coupling constant. Hence, it can be concluded that the isomeric form of vinylic group is trans. Cyclization of the diketo compounds $\mathbf{7 a - j}$ was achieved via refluxing with ammonium acetate in acetic acid to afford the corresponding pyrazino derivatives 8a-j. The chemical structures of the isolated products 8a-j were confirmed by their spectral and microanalytical data. In IR spectra, the notable feature was the disappearance of the stretching vibrations of the carbonyl groups.In addition, they were confirmed by MS and microanalytical data.

\section{BIOLOGICAL ACTIVITY}

\section{In vitro cytotoxicity screening}

The in vitro cytotoxicity studies of the newly synthesized compounds were performed on three different cancer cell lines, namely human hepatoma cell line (HepG2), human breast cancer cell line (MCF-7) and kidney of African green monkey (Vero B). The quantitative evaluation of the cytotoxicity screening was done by employing tetrazolium salt MTT (3-(4,5-dimethyl-2-thiazolyl)2,5-diphenyl-2H-tetrazolium bromide) assay. MTT assay is applied to assess cytotoxicity and viability of the cells. It is a colorimetric metabolic activity assay that measures the ability of viable cells to reduce a yellow tetrazolium salt to purple insoluble formazan in the mitochondria. Hence, formation of the purple formazan depends on the metabolic activity of the cells and directly related to the number of viable cells. However, the cytotoxicity of the tested compounds affects the metabolic activity of the cells and in turn, decreases the production of formazan. By comparing the amount of the formazan resulted from cells treated with the tested compounds with the amount of formazan resulted from untreated control cells, the cytotoxic effects of the target compounds can be concluded. The coloured formazan solution was measured using a spectrophotometer and the correspondent 
compound concentrations were calculated. Linear regression analysis was used to define dose-response curves and to compute the concentration of the tested compounds needed to reduce absorbance of the formazan by $50 \%$. So, the cytotoxicity of the tested compounds was expressed as the concentration that resulted in a $50 \%$ inhibition of growth $\left(\mathrm{IC}_{50}\right)$ compared to the untreated cells (DMSO without the tested compounds) and 5- FU as shown in Table 1.

Table 1: In vitro antitumor activities of the designed compounds.

\begin{tabular}{|c|c|c|c|}
\hline \multirow{2}{*}{ Compound No. } & \multicolumn{3}{|c|}{$\mathrm{IC}_{50}(\mu \mathrm{g} / \mathrm{ml})^{\mathrm{a}}$} \\
\hline & HepG2 $^{\text {b }}$ & MCF $-7^{c}$ & $\operatorname{Vero~}^{\mathrm{d}}$ \\
\hline $5 a$ & 2 & 1.8 & 3.5 \\
\hline $5 b$ & $\mathrm{ND}^{\mathrm{e}}$ & $\mathrm{ND}^{\mathrm{e}}$ & $\mathrm{ND}^{\mathrm{e}}$ \\
\hline $5 c$ & 5.5 & 2 & 2.5 \\
\hline $5 d$ & 4 & 3 & 4 \\
\hline $5 \mathrm{e}$ & 7 & 5.5 & 9 \\
\hline $5 f$ & 20 & 25 & 20 \\
\hline $7 \mathrm{a}$ & Inactive $^{f}$ & 20 & Inactive $^{f}$ \\
\hline $7 \mathrm{~b}$ & 30 & 2 & 2.5 \\
\hline $7 \mathrm{c}$ & $\mathrm{ND}^{\mathrm{e}}$ & $\mathrm{ND}^{\mathrm{e}}$ & $\mathrm{ND}^{\mathrm{e}}$ \\
\hline $7 d$ & $\mathrm{ND}^{\mathrm{e}}$ & $\mathrm{ND}^{\mathrm{e}}$ & $\mathrm{ND}^{\mathrm{e}}$ \\
\hline $7 \mathrm{e}$ & 30 & 10 & 10 \\
\hline $7 f$ & 20 & 20 & 20 \\
\hline $7 g$ & 30 & 20 & 20 \\
\hline $7 \mathrm{~h}$ & 30 & 20 & Inactive $^{\mathrm{f}}$ \\
\hline $7 \mathrm{i}$ & 20 & 7 & 10 \\
\hline $7 \mathrm{j}$ & 30 & 20 & 20 \\
\hline $8 a$ & Inactive $^{f}$ & 18 & 20 \\
\hline $8 \mathrm{~b}$ & 18 & 10 & 18 \\
\hline $8 \mathrm{c}$ & $\mathrm{ND}^{\mathrm{e}}$ & $\mathrm{ND}^{\mathrm{e}}$ & $\mathrm{ND}^{\mathrm{e}}$ \\
\hline $8 d$ & $\mathrm{ND}^{\mathrm{e}}$ & $\mathrm{ND}^{\mathrm{e}}$ & $\mathrm{ND}^{\mathrm{e}}$ \\
\hline $8 \mathrm{e}$ & 20 & 10 & 20 \\
\hline $8 \mathrm{f}$ & $\mathrm{ND}^{\mathrm{e}}$ & $\mathrm{ND}^{\mathrm{e}}$ & $\mathrm{ND}^{\mathrm{e}}$ \\
\hline $8 g$ & Inactive $^{f}$ & Inactive $^{f}$ & Inactive $^{\mathrm{f}}$ \\
\hline $8 \mathrm{~h}$ & 18 & 20 & 18 \\
\hline $8 \mathrm{i}$ & Inactive $^{f}$ & Inactive $^{\mathrm{f}}$ & Inactive $^{f}$ \\
\hline $8 \mathrm{j}$ & Inactive $^{f}$ & 20 & Inactive $^{f}$ \\
\hline $5-\mathrm{FU}^{\mathrm{g}}$ & 62 & 12 & 13 \\
\hline $\begin{array}{l}{ }^{\mathrm{a}} \mathrm{IC}_{50} \text { : Compound } \\
50 \% \\
{ }^{\mathrm{b}} \text { Human Hepatoce } \\
{ }^{\mathrm{c}} \text { Human breast ade } \\
{ }^{\mathrm{d}} \text { Kidney of normal } \\
{ }^{\mathrm{e}} \text { Not determined d } \\
\text { Inactive because t } \\
{ }^{\mathrm{g}} \text { 5-FU: } 5 \text {-Fluorour }\end{array}$ & $\begin{array}{l}\text { ntration re } \\
\text { carcinoma } \\
\text { cinoma cel } \\
\text { African gr } \\
\text { olubility p } \\
0 \text { values w }\end{array}$ & bit tum & iferatio \\
\hline
\end{tabular}

For the preliminary characterization of the expected cytotoxicity of the tested compounds, we first examined the changes in cell morphology induced by the treatment under a phase contrast microscope. The purple formazan should be visible inside the cells before dissolving it in isopropanol/ $\mathrm{HCl}$ solution. A qualitative morphological study of cytotoxicity by MTT assay was applied for compounds $\mathbf{5 c}$ and $\mathbf{7 i}$ using high and low doses and compared with the untreated cells (negative control). Viable cells exhibited deep purple cell nucleus with rod shaped formazon whereas apoptotic cells lacked formazon growth in a dose dependent manner (Figures 1 and 2).

Among the tested compounds, compounds 5a and 5c-e showed the highest and broad spectrum activity against the three cancer cell lines. Compound 5a, with unsubstituted phenyl ring, exploited great potency and lethal effect over HepG2, MCF-7 and Vero $\mathrm{B}$ cell lines with $\mathrm{IC}_{50}$ values of $2,1.8$ and $3.5 \mu \mathrm{g} / \mathrm{ml}$, respectively. It is clear that compound 5a $(1.8-3.5 \mu \mathrm{g} / \mathrm{ml})$ is almost 7 to 18 folds as 5-FU (12-62 $\mu \mathrm{g} / \mathrm{ml})$. By comparing the most active compound 5a with the other members in the same series, we concluded that, substitution of the phenyl ring or replacing it with a pyridyl ring relatively decreased the activity. With regard to the selectivity against individual cell lines, compound $\mathbf{5 a}$ showed selective inhibition against HepG2 and MCF-7 cell lines. However, compound $\mathbf{5 c}$ showed effectiveness against Vero B cell line with $\mathrm{IC}_{50}$ reached $2.5 \mu \mathrm{g} / \mathrm{ml}$. The results of the cytotoxicity of

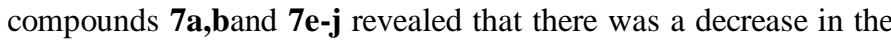
activity when compared with $\mathrm{N}$-unsubstituted compounds 5. Compound $\mathbf{7 b}$ showed promising antiproliferative activity against MCF-7 and Vero B cell lines at $\mathrm{IC}_{50}$ values of 2 and $2.5 \mu \mathrm{g} / \mathrm{ml}$, respectively. In addition, both $7 \mathbf{e}$ and $7 \mathbf{i}$ showed good growth inhibition against MCF-7 and Vero B cell lines, while the rest of compounds among this series exerted moderate activity with $\mathrm{IC}_{50}$ ranging from 20-30 $\mu \mathrm{g} / \mathrm{ml}$. However, compound 7a showed activity against only MCF-7 cell line, while compound $\mathbf{7 h}$ gave its activity against only HepG2 and MCF-7 cell lines. A notable decrease in the inhibitory activity was observed in the cyclized pyrazino derivative $\mathbf{8 b}$ against MCF-7 and Vero B cell lines at $\mathrm{IC}_{50}$ 10 and $18 \mu \mathrm{g} / \mathrm{ml}$, respectively in comparison with the activity of its open chain analogue $7 \mathbf{b}$ against the same cell lines $\left(\mathrm{IC}_{50} 2\right.$ and 2.5 $\mu \mathrm{g} / \mathrm{ml}$, respectively). On the other hand, a little or no change in the activity of most of the cyclized pyrazino derivatives was observed in comparison with that of the corresponding uncyclized $\alpha, \beta$ unsaturated ketone derivatives $\mathbf{7}$.

The active pyrazino compounds showed good $\mathrm{IC}_{50}$ in the range of $10-20 \mu \mathrm{g} / \mathrm{ml}$. The increased inhibitory activity of compounds $\mathbf{8 b}, \mathbf{8 h}$ and $\mathbf{8 j}$ in comparison to compounds $8 \mathbf{a}, \mathbf{8 g}$ and $\mathbf{8 i}$, respectively, may reflect the positive impact of the lipophilicity of the molecules resulted from replacement of $\mathrm{Cl}$ by $\mathrm{Br}$ atom. However, compounds $\mathbf{8 g}$ and $\mathbf{8 i}$ were completely inactive against all of the three cell lines tested.
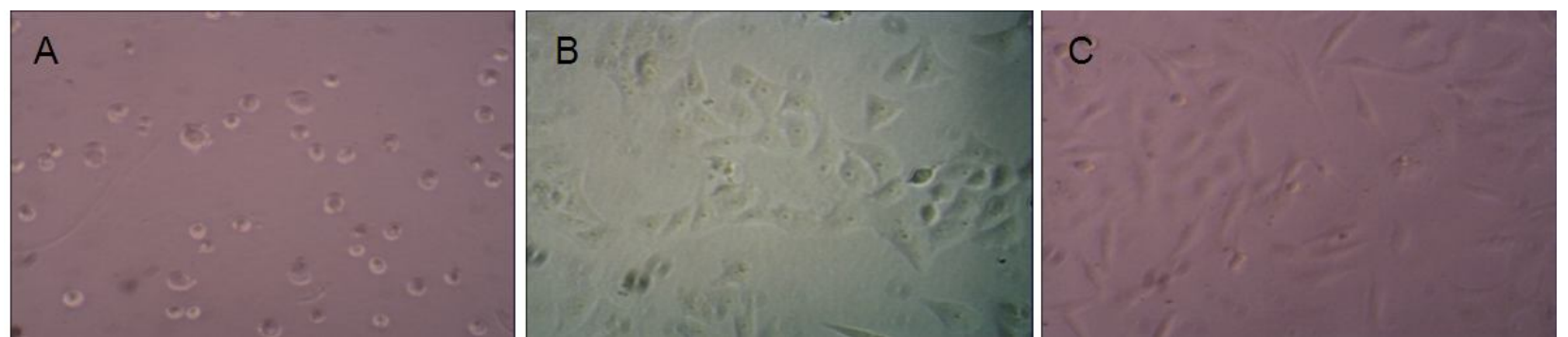

Fig. 1: A-C Representative phase micrograph of MCF-7 of the control and of those treated cells with 5c. (A) MCF-7 treated (37 $\mu \mathrm{g} / \mathrm{ml})(\mathrm{B}) \mathrm{MCF}-7 \mathrm{treated}(4$ $\mu \mathrm{g} / \mathrm{ml})$ and (C) Control cells, untreated MCF-7 cells. 


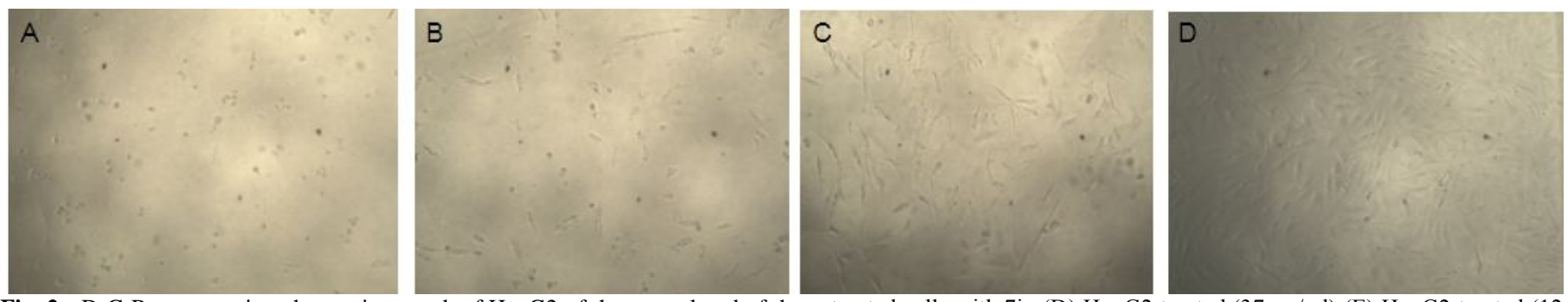

Fig. 2: D-G Representative phase micrograph of HepG2 of the control and of those treated cells with 7i. (D) HepG2 treated (37 $\mu \mathrm{g} / \mathrm{ml})(\mathrm{E}) \mathrm{HepG} 2 \mathrm{treated}(12$ $\mu \mathrm{g} / \mathrm{ml})(\mathrm{F}) \mathrm{HepG} 2$ treated $(4 \mu \mathrm{g} / \mathrm{ml})$ and $(\mathrm{G})$ Control cells, untreated HepG2 cells.

\section{LIPINSKI'S RULE OF FIVE}

Lipinski's rule of five provided very convenient and easily applied guidelines for the selection of compounds with a greater chance of yielding successful drugs that are orally active in human (Lipinski et al., 1997). If the rule of five score is greater than one, the compound is unlikely to be further pursued as a potential drug, because it would likely lack properties important in its ADME (Lipinski, 2004). The results showed that all investigated compounds obeyed these rules and should present good passive oral absorption (Table 2).

Table 2: Calculated Lipinski's rule of five for the biologically active compounds

\begin{tabular}{cccccc}
\hline \multirow{2}{*}{$\begin{array}{c}\text { Comp. } \\
\text { No. }\end{array}$} & M.wt $^{\mathbf{a}}$ & $\log ^{\mathbf{b}}$ & $\mathbf{H B D}^{\mathbf{c}}$ & HBA $^{\mathbf{d}}$ & $\begin{array}{c}\text { No. of } \\
\text { violations }\end{array}$ \\
\cline { 2 - 6 } $\mathbf{5 a}$ & 248.28 & 3.408 & 1 & 3 & 0 \\
$\mathbf{5 c}$ & 316.28 & 4.304 & 1 & 3 & 0 \\
$\mathbf{5 d}$ & 266.28 & 3.572 & 1 & 3 & 0 \\
$\mathbf{5 e}$ & 249.27 & 1.992 & 1 & 4 & 0 \\
$\mathbf{5 f}$ & 249.27 & 2.119 & 1 & 4 & 0 \\
$\mathbf{7 a}$ & 430.88 & 5.358 & 0 & 5 & 1 \\
$\mathbf{7 b}$ & 475.34 & 5.489 & 0 & 5 & 1 \\
$\mathbf{7 e}$ & 418.86 & 5.465 & 0 & 4 & 1 \\
$\mathbf{7 f}$ & 463.31 & 5.596 & 0 & 4 & 1 \\
$\mathbf{7 g}$ & 401.85 & 3.885 & 0 & 5 & 0 \\
$\mathbf{7 h}$ & 446.30 & 4.017 & 0 & 5 & 0 \\
$\mathbf{7 i}$ & 401.85 & 4.012 & 0 & 5 & 0 \\
$\mathbf{7 j}$ & 446.30 & 4.143 & 0 & 5 & 0 \\
$\mathbf{8 a}$ & 411.88 & 6.927 & 0 & 4 & 1 \\
$\mathbf{8 b}$ & 456.34 & 7.058 & 0 & 4 & 1 \\
$\mathbf{8 e}$ & 399.85 & 7.034 & 0 & 3 & 1 \\
$\mathbf{8 h}$ & 427.30 & 5.586 & 0 & 4 & 1 \\
$\mathbf{8 j}$ & 427.30 & 5.713 & 0 & 4 & 1 \\
\hline
\end{tabular}

${ }^{a}$ Molecular weight.

${ }^{b}$ An octanol-water partition coefficient

${ }^{\mathbf{c}}$ Number of hydrogen bond donors.

${ }^{\mathrm{d}}$ Number of hydrogen bond acceptor.

\section{DOCKING STUDIES}

The promising antiproliferative activities of compounds 5a and7b over breast cancer cells stimulated us to study the molecular docking of these compounds into the active site of EGFR, which is over expressed in breast cancer cells. The aim of this study is to reveal if these compounds have comparable binding manner to EGFR inhibitors such as Erlotinib. We assumed that the active target compounds $\mathbf{5 a}$ and $\mathbf{7 b}$ might demonstrate antiproliferative activity against MCF-7 cell line through inhibition of EGFR. The automated docking program of MOE 2008.10 was used to dock compounds $\mathbf{5 a}$ and7 $\mathbf{b}$ into the active binding site of EGFR along with the inhibitor Erlotinib. The calculated binding energies of the docked compounds $\mathbf{5 a}, \mathbf{7 b}$ were $-82.10,-71.22$ and $-114.13 \mathrm{kj} / \mathrm{mol}$, respectively. The carbonyl group of compound 5a showed two hydrogen bonds with Thr-766 $(2.98 \AA)$ and $\mathrm{HOH}-10 \quad(3.03 \AA)$ mediated hydrogen bonding

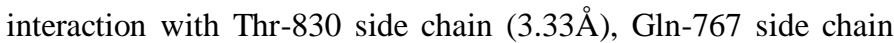

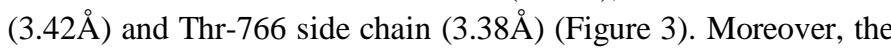
benzimidazole ring of compound $\mathbf{5 a}$ binds to a narrow hydrophobic pocket in the N-terminal domain of EGFR-TK similar to quinazoline ring of Erlotinib inhibitor. So, the results from the molecular docking study confirmed that the active compound 5a may act on the same target receptor as Erlotinib.On the other hand compound $7 \mathbf{b}$ bound with active binding site of EGFRin a different mode of interaction in which the carbonyl groups of both chalcone and phenacyl moieties showed one hydrogen bond with HOH-10 (2.66 and 3.81 bonding interaction with Thr-766 side chain (2.71 $\mathrm{A}$ ) (Figure 3). . This different mode may be attributed to steric clash of the phenacyl moiety during docking procedure.
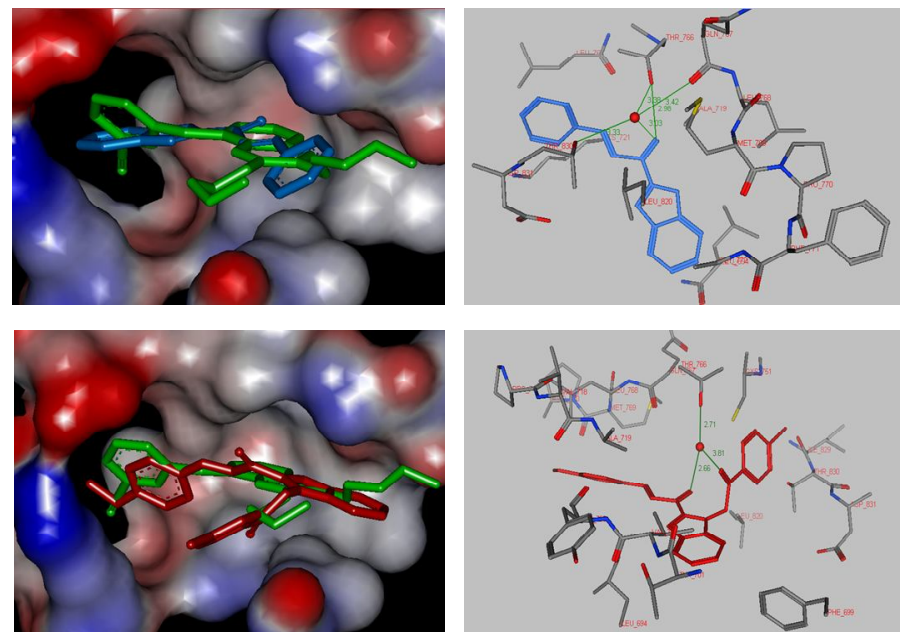

Fig. 3: The binding model of compounds 5a (Upper panel in blue) and $7 \mathrm{~b}$ (Lower panel in red) with EGFR TK complex (PDB ID: 1M17). Left upper and lower panels showed overlay of the docked $5 \mathrm{a}$ and $7 \mathrm{~b}$ with Erlotinib ligand (in green). The hydrogen bonds are shown in green lines. 


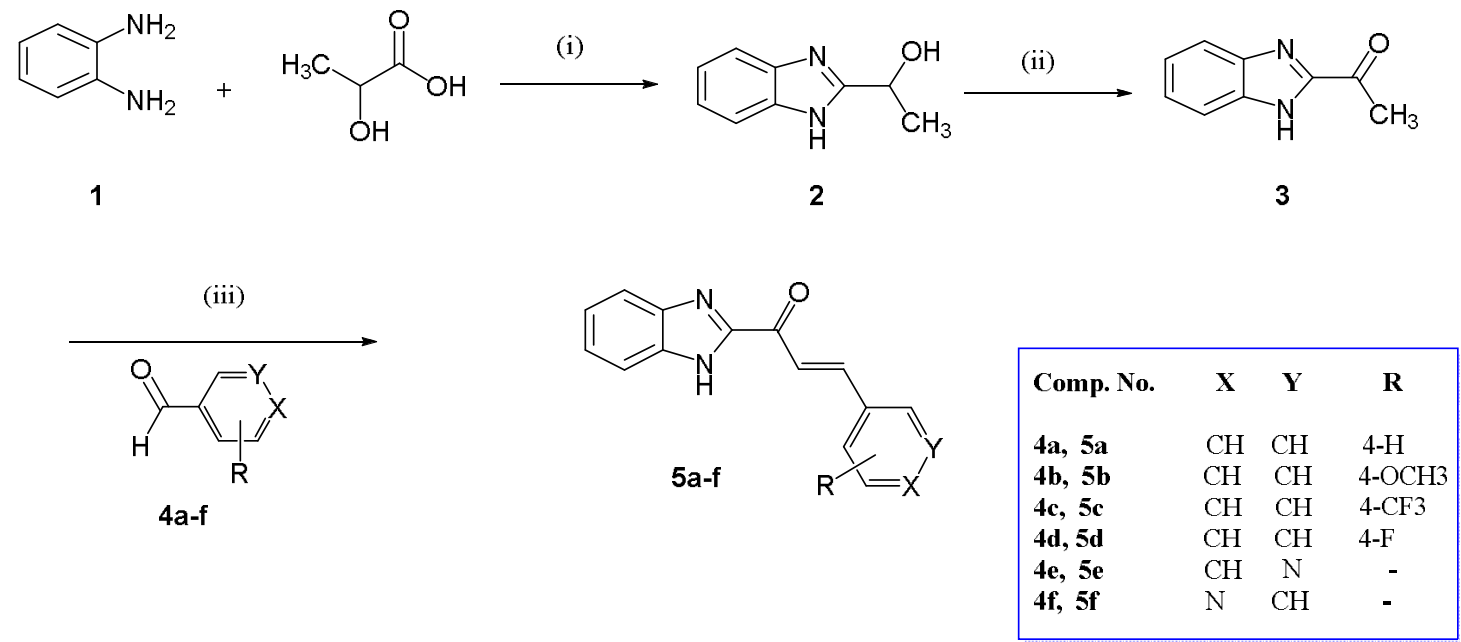

Scheme 1 Reaction protocol for the synthesis of 2, 3 and 5a-f: (i) $4 \mathrm{~N} \mathrm{HCl}$; (ii) $\mathrm{K}_{2} \mathrm{Cr}_{2} \mathrm{O}_{7} / \mathrm{H}_{2} \mathrm{SO}_{4}$; (iii) Ethanol/ $\mathrm{H}_{2} \mathrm{O}, 10 \% \mathrm{NaOH}$

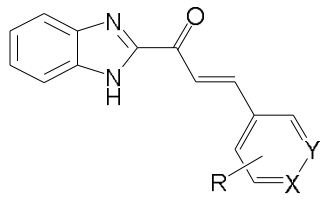

5a-f

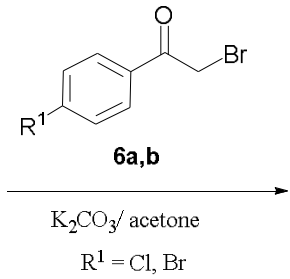

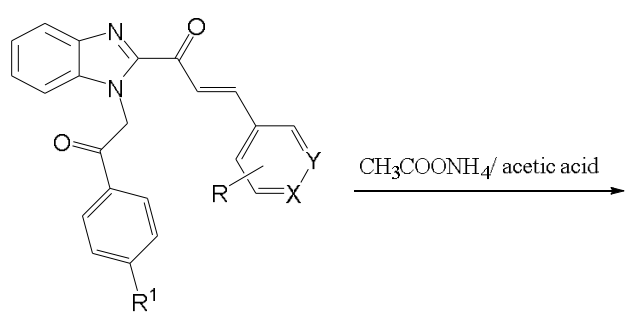

$7 \mathrm{a}-\mathrm{j}$

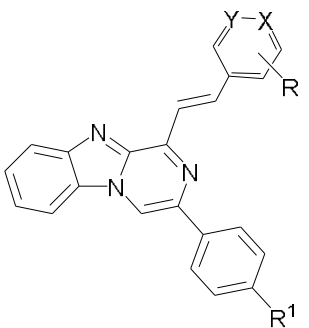

$8 \mathrm{a}-\mathrm{j}$

$\begin{array}{lccll}\text { Comp.No. } & \mathbf{X} & \mathbf{Y} & \mathbf{R} & \mathbf{R}^{\mathbf{1}} \\ \mathbf{7 a}, \mathbf{8 a} & \mathrm{CH} & \mathrm{CH} & 4-\mathrm{OCH} 3 & \mathrm{Cl} \\ \mathbf{7 b}, \mathbf{8 b} & \mathrm{CH} & \mathrm{CH} & 4-O C H 3 & \mathrm{Br} \\ \mathbf{7 c , 8}, \mathbf{8} & \mathrm{CH} & \mathrm{CH} & 4-\mathrm{CF} 3 & \mathrm{Cl} \\ \mathbf{7 d}, \mathbf{8 d} & \mathrm{CH} & \mathrm{CH} & 4-\mathrm{CF} 3 & \mathrm{Br} \\ \mathbf{7 e , 8 e} & \mathrm{CH} & \mathrm{CH} & 4-\mathrm{F} & \mathrm{Cl} \\ \mathbf{7 f}, \mathbf{8 f} & \mathrm{CH} & \mathrm{CH} & 4-\mathrm{F} & \mathrm{Br} \\ \mathbf{7 g}, \mathbf{8 g} & \mathrm{CH} & \mathrm{N} & - & \mathrm{Cl} \\ \mathbf{7 h}, \mathbf{8 h} & \mathrm{CH} & \mathrm{N} & - & \mathrm{Br} \\ \mathbf{7 i}, \mathbf{8 i} & \mathrm{N} & \mathrm{CH} & - & \mathrm{Cl} \\ \mathbf{7 j}, \mathbf{8} \mathbf{j} & \mathrm{N} & \mathrm{CH} & - & \mathrm{Br}\end{array}$

Scheme 2 Reaction protocol for the synthesis of 7, 8a-j: (i) $\mathrm{K}_{2} \mathrm{CO}_{3} /$ acetone; (ii) $\mathrm{CH}_{3} \mathrm{COONH}_{4} /$ acetic acid.

\section{CONCLUSION}

A series of substituted benzimidazole and pyrazinobenzimidazole derivatives were developed and three cancer cell lines including HepG2, MCF-7 and Vero B were used to evaluate the cytotoxic activity of these designed compounds. Among the tested compounds, Compounds 5a and 5c-e showed the highest and broad spectrum activity against the three cancer cell lines with $\mathrm{IC}_{50}$ range of $1.8-9 \mu \mathrm{g} / \mathrm{ml}$, comparable to 5-FU with $\mathrm{IC}_{50}$ range of $12-62 \mu \mathrm{g} / \mathrm{ml}$. Compound 5a, with unsubstituted phenyl ring, free benzimidazole $\mathrm{NH}$ and $\alpha, \beta$-unsaturated carbonyl moiety at the 2-position of benzimidazole nucleus, exploited great potency and lethal effect over HepG2, MCF-7 and Vero B cell lines with $\mathrm{IC}_{50}$ values of $2,1.8$ and $3.5 \mu \mathrm{g} / \mathrm{ml}$, respectively. Molecular docking studies confirmed the strong cytotoxic activity of compounds 5a and 7b over MCF-7 and the postulation that these active compounds may act on the same enzyme target where EGFR inhibitor, Erlotinib, acts.

\section{ACKNOWLEDGMENTS}

Our honest appreciation to the Chemical Computing Group Inc, 1010 Sherbrooke Street West, Suite 910, Montreal, H3A 2R7, Canada., for its agreement for using MOE 2008.10 software.

\section{Financial support and sponsorship: Nil.}

Conflict of Interests: There are no conflicts of interest.

\section{REFERENCES}

Abdel-Mohsen HT, Ragab FAF, Ramla MM, El Diwani HI. Novel benzimidazole-pyrimidine conjugates as potent antitumor agents. Eur J Med Chem, 2010; 45: 2336-2344.

Bagi CM. Summary-Cancer cell metastasis session. J Musculoskel Neuron Interact, 2002; 2: 579-580

Balmer CM, Valley AW.Cancer Treatment and Chemotherapy; Pharmacotherapy A Pathophysiologic Approach, (5th Ed.), (Dipiro JT, 
Talbert RL, Matzke GR, Wells BG, Posey LM. (1stEd.), McGraw-Hill, USA, 2002; 2175-222.

Bansal Y, Silakari O.The therapeutic journey of benzimidazoles. Bioorg Med Chem, 2012; 1-29.

Boschelli DH, Denny WA, Doherty AM, Hamby JM, Khatana SS, Kramer JB, Palmer BD, Showalter HDH. Benzimidazoles for inhibiting protein tyrosine kinase mediated cellular proliferation, US patent No. 5,990,146, 1999; November 23.

Cockerill GS, Lackey KE.Small molecule inhibitor of the class I receptor tyrosine kinase family.Curr Top Med Chem, 2002; 2: 1001-1010.

Cockerill S, Stubberfield C, Stables J, Carter M, Guntrip S, Smith K, McKeown S, Shaw R, Topley P, Thomsen L, Affleck K, Jowett A, Hayes D, Willson M, Woollard P, Spalding D. Indazolylaminoquinazolinesand pyridopyrimidines as inhibitors of the EGFr and C-erbB-2.Bioorg Med ChemLett, 2001; 11(11): 1401-1405.

Denizot, F.; Lang, R. Rapid colorimetric assay for cell growth and survival. Modifications to the tetra-zolium dye procedure giving improved sensitivity and reliability. J. Immunol. Methods 1986, 89, 271277.

El-Azab AS, Al-Omar MA, Abdel-Aziz AA-M, Abdel-Aziz NI, El-Sayed MA-A, Aleisa AM, Sayed-Ahmed MM, Abdel-Hamide SG. Design, synthesis and biological evaluation of novel quinazoline derivatives as potential antitumor agents: Molecular docking study. Eur J Med Chem, 2010; 45: 4188-4198.

Gowda NRT, Kavitha CV, Chiruvella KK, Joy O, Rangappa KS, Raghavan SC. Synthesis and biological evaluation of novel 1-(4methoxyphenethyl)-1H-benzimidazole-5-carboxylic acid derivatives and their precursors as antileukemic agents. Bioorg Med ChemLett, 2009; 19: 4594-4600.

Halgren TA.Merck molecular force field. 1. Basis, form, scope, parameterization, and performance of MMFF94. J ComputChem, 1996; 17: 490-519.

Hamed MM, Abou El Ella DA, Keeton AB, Piazza GA, Engel M, Hartmann RW, Abadi AH.Quinazoline and tetrahydropyridothieno[2,3-d]pyrimidine derivatives as irreversible EGFR tyrosine kinase inhibitors: influence of the position 4 substituent. Med ChemCommun, 2013; 4: 1202-1207.

Hida F, Robert J, Luu-Duc C. Synthesis and evaluation of benzimidazole and imidazole compounds as potential aromatase inhibitors. Farmaco, 1994; 49: 489-492.

Hranjec M, Starcevic K, Pavelic SK, Lucin P, Pavelic K, Zamola GK. Synthesis, spectroscopic characterization and antiproliferative evaluation in vitro of novel Schiff bases related to benzimidazoles. Eur J Med Chem, 2011; 46: 2274-2282.

Kontoyianni M, McClellan LM, Sokol GS. Evaluation of docking performance: comparative data on docking algorithms. J Med Chem, 2004; 47: 558-565.

Lipinski CA. Lead- and drug-like compounds: the rule-of-five revolution. Drug Discovery Today Technologies, 2004; 1 (4): 337-341.

Lipinski CA, Lombardo F, Dominy BW, Feeney PJ.Expeimental and computational approaches to estimate solubility and permeability in drug discovery and development settings.Adv Drug Del Rev, 1997; 23: 3-25.

Luo Y, Xiao F, Qian S, Lu W, Yang B. Synthesis and in vitro cytotoxic evaluation of some thiazolylbenzimidazole derivatives. Eur J Med Chem, 2011; 46: 417-426.

MOE 2008.10 of Chemical Computing Group.Inc.

Mosmann T. Rapid colorimetric assay for cellular growth and survival: application to proliferation and cytotoxicity assays. J Immunol Methods, 1983; 65 (1-2): 55-63.

Neff DK, Dutra AL, Blevitt JM, Axe FU, Hack MD, Buma JC, Rynberg R, Brunmark A, Karlsson L. 2-Aryl benzimidazoles featuring alkyl-linked pendant alcohols and amines as inhibitors of checkpoint kinase Chk2.Bioorg Med ChemLett, 2007; 17: 6467-6471.

Ng RA, Guan J, Alford VC, Lanter JC, Allan GF, Sbriscia T, Lundeen SG, Sui Z. 2-(2,2,2-Trifluoroethyl)-5,6-dichlorobenzimidazole derivatives as potent androgen receptor antagonists. Bioorg Med ChemLett, 2007; 17: 955-958.
Pool WO, Harwood HJ, Ralston AW.2-Alkyl benzimidazoles as derivatives for identification of aliphatic acids. J AmerChemSoc, 1937; 1: 178-179.

RahmanMdA, Siddiqui AA.Pyrazoline derivatives: A worthy insight into the recent advances and potential pharmacological activities. Int J Pharm Sci Drug Res, 2010; 2 (3):165-175.

Ramla MM, Omar MA, El-Khamry AMM, El-Diwani HI.Synthesis and antitumor activity of 1-substituted-2-methyl-5nitrobenzimidazoles.Bioorg Med Chem, 2006; 14: 7324-7332.

Ramla MM, Omar MA, Tokuda H, El-Diwani HI. Synthesis and inhibitory activity of new benzimidazole derivatives against Burkitt's lymphoma promotion. Bioorg Med Chem, 2007; 15, 6489-6469.

Refaat HM. Synthesis and anticancer activity of some novel 2substituted benzimidazole derivatives.Eur J Med Chem, 2010; 45: 29492956.

Selvakumar S, Babu IS, Chidambaranathan N. Pharmacological evaluation of some potent 2-substituted benzimidazolylchalcones for analgesic, anti-inflammatory, anthelmintic and central nervous system activities. International Journal of Phytopharmacology, 2012; 3: 163-172.

Shaharyar M, Abdullah MM, Bakht MA.Pyrazoline bearing benzimidazoles: Search for anticancer agents. Eur J Med Chem, 2010; 45: 114-119.

Silverstein RM, Bassler GC, Morrill TC.1991, Spectroscopic Identification of Organic Compounds, John Wiley and sons (5th Ed.), Inc. NY.

Soni Y, Deepa S, SanjayKS, Vinay KS.Novel BenzimidazoleAnalogs as Inhibitors of EGFR Tyrosine Kinase.ChemBiol Drug Des, 2012; 80: 625-630.

Su, Z; Yang, Z.; Xu,Y.; Chen,Y.; Yu, Q.. Apoptosis, autophagy, necroptosis, and cancer metastasis. Molecular Cancer 2015, 48, 1-14.

Stamos, J.; Sliwkowski, M. X.; Eigenbrot, C. Structure of the epidermal growth factor receptor kinase domain alone and in complex with a 4-anilinoquinazoline inhibitor.J. Biol. Chem. 2002, 277, 4626546272.

Styskala J, Styskalova L, Hajduch M. Synthesis of 2-aryl-4 (benzimidazol-2-yl)-1,2-dihydro[1,2,4]triazino[4,5-a]benzimidazol-1-one derivatives with preferential cytotoxicity against carcinoma cell lines. Eur J Med Chem, 2008;43: 449-455.

Thimmegowda NR, Swamy SN, Kumar CSA, Kumar YCS, Chandrappa S, Yip GW, Rangappa KS. Synthesis, characterization and evaluation of benzimidazole derivative and its precursors as inhibitors of MDA-MB-231 human breast cancer cell proliferation. Bioorg Med ChemLett, 2008; 18: 432-435.

Vogelstein, B.; Papadopoulos, N.; Velculescu, V. E.; Zhou, S.; Diaz, L. A.; Kinzler, K. W.; Cancer genome landscapes, Science, 2013, 339(6127), 1546-1558.

Wakeling AE.Epidermal growth factor receptor tyrosine kinase inhibitors.CurrOpinPharmacol, 2002; 2: 382-387.

Zhong M, Bui M, Shen W, Baskaran S, Allen DA, Elling RA, Flanagan WM, Fung AD, Hanan E J, Harris SO, Heumann SA, Hoch U, Ivy SN, Jacobs JW, Lam S, Lee H, McDowell R S, Oslob JD, Purkey HE, Romanowski MJ, Silverman JA, Tangonan BT, Taverna P, Yang W, Yoburn JC, Yu CH, Zimmerman KM, Brien TO, Lew W. 2Aminobenzimidazoles as potent Aurora kinase inhibitors. Bioorg Med Chem Lett, 2009; 19: 5158-5161.

\section{How to cite this article:}

Mohamed AAB, Badria FA, Maarouf AR, Abdel-Aziz NI, ElSenduny F, Abdel-Aziz AM, Bayomi SM. Synthesis, antitumor evaluation and molecular modeling study of novel benzimidazoles and pyrazinobenzimidazoles. J App Pharm Sci, 2017; 7 (06): 206214. 